\title{
The flexible asymmetric shock tube (FAST): a Ludwieg tube facility for wave propagation measurements in high-temperature vapours of organic fluids
}

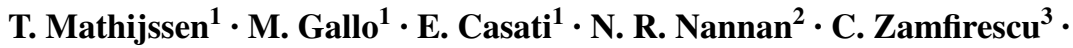 \\ A. Guardone ${ }^{4} \cdot$ P. Colonna ${ }^{1}$
}

Received: 11 March 2015 / Revised: 2 September 2015 / Accepted: 8 September 2015 / Published online: 5 October 2015

(C) The Author(s) 2015. This article is published with open access at Springerlink.com

\begin{abstract}
This paper describes the commissioning of the flexible asymmetric shock tube (FAST), a novel Ludwieg tube-type facility designed and built at Delft University of Technology, together with the results of preliminary experiments. The FAST is conceived to measure the velocity of waves propagating in dense vapours of organic fluids, in the so-called non-ideal compressible fluid dynamics (NICFD) regime, and can operate at pressures and temperatures as high as 21 bar and $400{ }^{\circ} \mathrm{C}$, respectively. The set-up is equipped with a special fast-opening valve, separating the high-pressure charge tube from the lowpressure plenum. When the valve is opened, a wave propagates into the charge tube. The wave speed is measured using a time-of-flight technique employing four pressure transducers placed at known distances from each other. The first tests led to the following results: (1) the leakage rate of $5 \times 10^{-4} \mathrm{mbarl} \mathrm{s}^{-1}$ for subatmospheric and $5 \times 10^{-2} \mathrm{mbar}^{-1} \mathrm{~s}^{-1}$ for a superatmospheric pressure is compatible with the purpose of the conceived experiments,
\end{abstract}

T. Mathijssen

T.Mathijssen@tudelft.nl

P. Colonna

P.Colonna@tudelft.nl

1 Propulsion and Power, Delft University of Technology, Kluyverweg 1, 2629 HS Delft, The Netherlands

2 Mechanical Engineering Discipline, Anton de Kom University of Suriname, Leysweg 86, PO Box 9212, Paramaribo, Suriname

3 Department of Automotive, Mechanical and Manufacturing Engineering, Faculty of Engineering and Applied Science, University of Ontario Institute of Technology, 2000 Simcoe St. N, Oshawa, ON L1H 7K4, Canada

4 Department of Aerospace Science and Technology, Politecnico di Milano, Via La Masa 34, 20156 Milan, Italy
(2) the process start-up time of the valve has been found to be between 2.1 and $9.0 \mathrm{~ms}$, (3) preliminary rarefaction wave experiments in the dense vapour of siloxane $\mathrm{D}_{6}$ (dodecamethylcyclohexasiloxane, an organic fluid) were successfully accomplished up to temperatures of $300^{\circ} \mathrm{C}$, and (4) a method for the estimation of the speed of sound from wave propagation experiments is proposed. Results are found to be within $2.1 \%$ of accurate model predictions for various gases. The method is then applied to estimate the speed of sound of $\mathrm{D}_{6}$ in the NICFD regime.

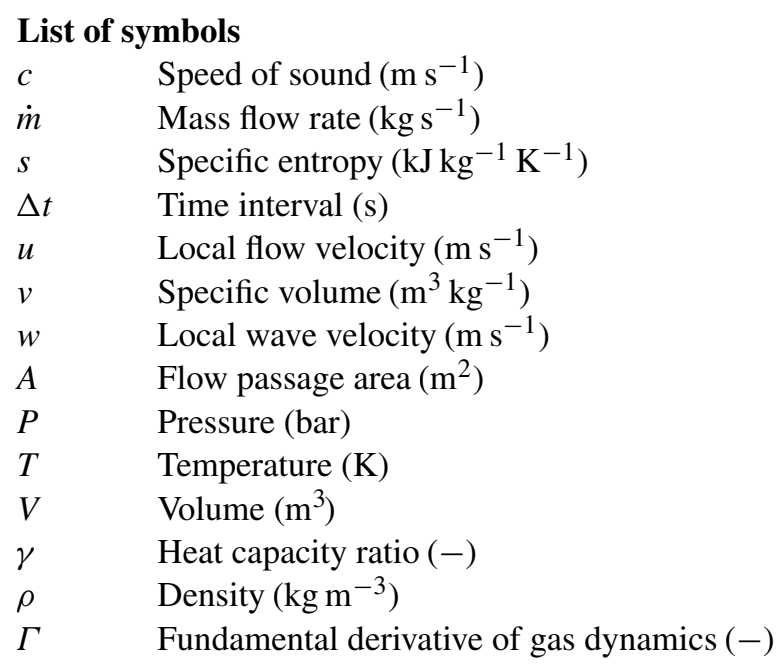

Acronyms

CT Charge tube

$\mathrm{D}_{6} \quad$ Dodecamethylcyclohexasiloxane

FAST Flexible asymmetric shock tube

FOV Fast-opening valve

LPP Low-pressure plenum

LR Leak rate (mbar $\left.1 \mathrm{~s}^{-1}\right)$

MW Molecular weight $\left(\mathrm{g} \mathrm{mol}^{-1}\right)$ 


$\begin{array}{ll}\text { NICFD } & \text { Non-ideal compressible fluid dynamics } \\ \text { ORC } & \text { Organic rankine cycle } \\ \text { PAC } & \text { Programmable automation controller } \\ \text { PID } & \text { Proportional-integral-derivative } \\ \text { RT } & \text { Reference tube } \\ \text { RW } & \text { Rarefaction wave } \\ \mathrm{scCO}_{2} & \text { Supercritical carbon dioxide }\end{array}$

\section{Introduction}

Non-ideal compressible fluid dynamics (NICFD) encompasses the field of fluid mechanics studying the motion of fluids in the dense vapour, dense vapour-liquid and supercritical thermodynamic region.

Such flows are characterized by, among other interesting phenomena, a quite different variation of the sound speed compared to that of ideal gases. The fundamental derivative of gas dynamics $\Gamma$, defined by Thompson (1971) as

$\Gamma \equiv 1+\frac{\rho}{c}\left(\frac{\partial c}{\partial \rho}\right)_{s}$

is constant in ideal gases and equal to $(\gamma+1) / 2$, while NICFD occurs whenever $\Gamma$ is variable among the thermodynamic states of the considered fluid flow (Thompson 1988).

NICFD is encountered in a variety of industrial processes. Notable examples are the working fluid flows within turbomachinery of novel technologies for the conversion of renewable energy sources, like organic Rankine cycle (ORC) (Cramer 1989; Brown and Argrow 2000; Colonna et al. 2015) or supercritical carbon dioxide $\left(\mathrm{scCO}_{2}\right)$ (Conboy et al. 2012; Rinaldi et al. 2015) power systems and in high-temperature heat pumps (Zamfirescu and Dincer 2009).

However, measurements in NICFD flows are scarce (Nannan et al. 2007; Weith et al. 2014). Hence, flow measurements in the dense vapour region of complex organic fluids can contribute to the understanding of NICFD and to the improvement of fluid thermodynamic models.

Shock tubes are arguably the experimental device of choice for the study of NICFD flows, as they have been already successfully used for measurements of shocks and unsteady wave propagation in fluids. Examples include shock tubes for the study of non-equilibrium vapour condensation (Maerefat et al. 1989), droplet condensation in expansion tubes (Peters and Rodemann 1998) and studies of particle-dense flow fields (Wagner et al. 2012).

A shock tube experiment aimed at investigating NICFD flows has been pursued in the early 2000s at the University of Colorado at Boulder by Fergason et al. (2003). The working fluid was PP10 (perfluorofluorene, $\mathrm{C}_{13} \mathrm{~F}_{22}$ ), the design operating temperature $360{ }^{\circ} \mathrm{C}$ and the design pressure 15.5 bar. A configuration with a diaphragm was adopted. The experiment failed because the working fluid underwent thermal decomposition due to the high operating temperature and possibly to the lack of precautionary measures to avoid the presence of air and moisture within the fluid, which are known catalytic agents of thermal degradation in organic fluids (Dvornic 2004; Calderazzi and Colonna 1997; Pasetti et al. 2014). In addition, the repeatable rupture of the shock tube diaphragm proved critical due to the relatively small pressure difference and the large acoustic impedance of the fluid (Thompson and Loutrel 1973; Guardone 2007). Furthermore the duration of preparatory activities for the experiments was relatively long since the diaphragm had to be replaced after each test.

This work documents the flexible asymmetric shock tube (FAST) set-up and the first experimental results of wave propagation measurements in the dense vapour of dodecamethylcyclohexasiloxane $\left(\mathrm{D}_{6}\right)$. The FAST is an unconventional Ludwieg tube designed and installed at Delft University of Technology (Colonna et al. 2008a), in the Netherlands, with the aim of studying wave propagation in the dense vapour of organic compounds. Figure 1 shows a schematic overview of a rarefaction wave experiment. The charge tube (CT) is filled with the dense organic vapour and kept at the desired pressure and temperature. The fast-opening valve (FOV), initially closed, is opened, thus allowing the fluid to flow towards the low-pressure plenum (LPP), which is maintained at a lower pressure. Consequently, a rarefaction wave travels into the CT.

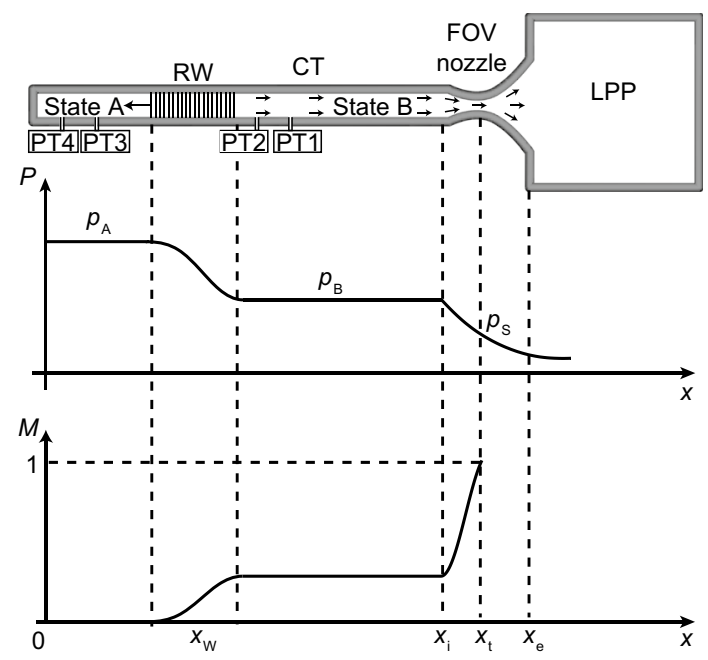

Fig. 1 Schematic overview of a rarefaction wave (RW) experiment. The upper charts show the qualitative pressure profile after the opening of the FOV separating the charge tube (CT) from the low-pressure plenum (LPP). The lower chart shows the Mach number of the flow in the laboratory frame. The fluid is accelerated from rest condition A to condition B and flows into the reservoir through the nozzle. At the nozzle throat, sonic conditions $\mathrm{S}$ are attained. The pressure is captured by pressure transducers PT1 to PT4 
A description of the set-up is provided in Sect. 2: it illustrates details about the components equipping the complete system as built, about the procedure of an experiment and an overview of the control and data acquisition system. The results of the characterization of the set-up, demonstrating the correct operation of the facility and validating the measurement systems, are reported and discussed in Sect. 3. In Sect. 4, results of preliminary rarefaction wave measurements in $\mathrm{D}_{6}$ are reported. Section 5 summarizes concluding remarks and outlines future work.

\section{The FAST and the experimental procedure}

The mechanical and heating equipment is described in Sect. 2.1 by outlining the procedure of a typical experiment. The measurement and monitoring instruments as well as the control system are briefly reported in Sect. 2.2.

\subsection{Equipment and procedure}

A schematic representation of the FAST is reported in Fig. 2 and a photograph in Fig. 3. All pipes, vessels and parts in contact with the working fluid are made of stainless steel (316Ti). Before starting an experiment, the working fluid undergoes a purification process to remove air and moisture as much as possible, which are known to promote decomposition at high temperature (Calderazzi and Colonna 1997; Pasetti et al. 2014). The facility is initially evacuated and closed off. The working fluid is introduced through pipe 1 into the vapour generator (Fig. 4), a custommade 5.9-litre vessel designed to heat and evaporate the working fluid up to the desired pressure under isochoric conditions. At the bottom of the vessel, the liquid can be extracted through a valve connected to flange F1.

In order to attain the desired pressure and temperature, the fluid is heated isochorically while enclosed in

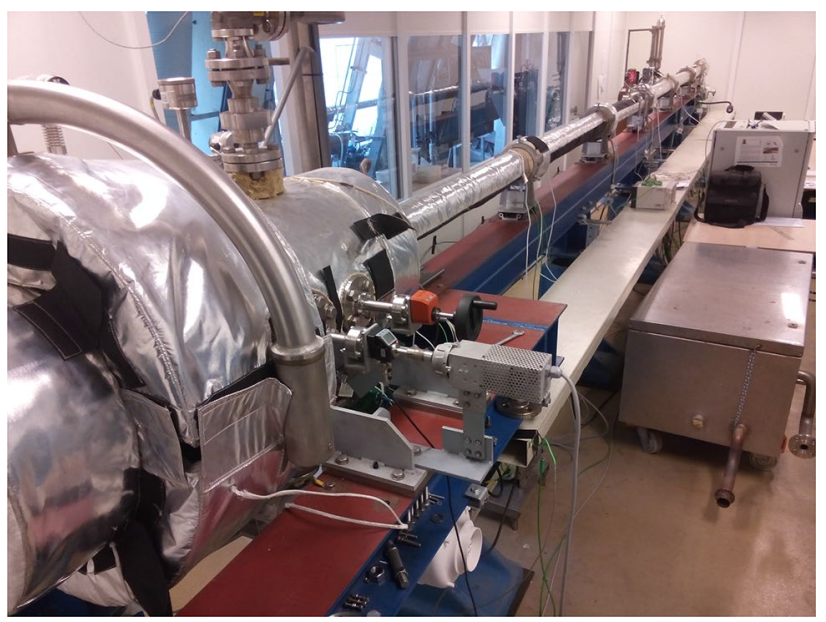

Fig. 3 Picture of the FAST set-up

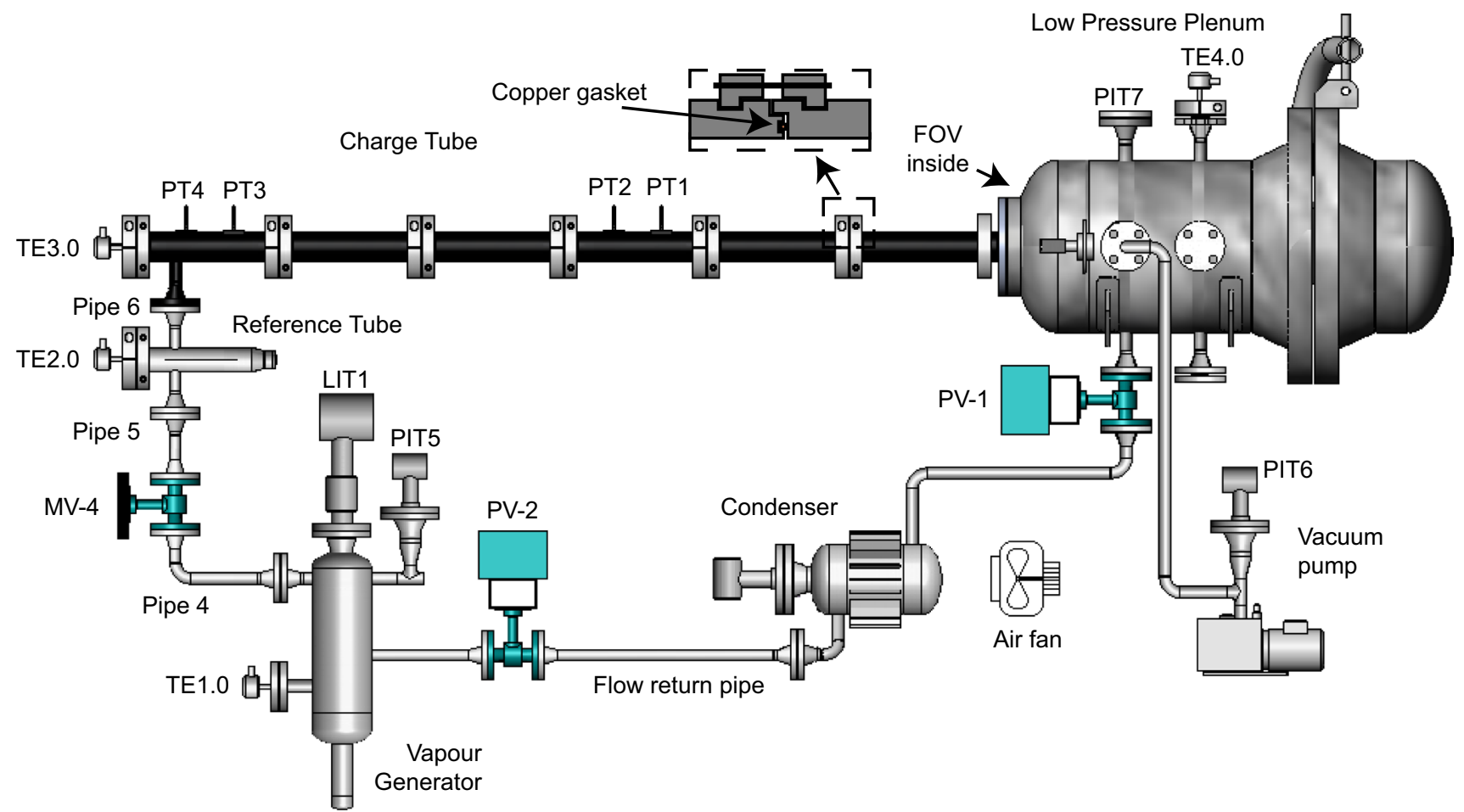

Fig. 2 Overview of the FAST set-up. The fast-opening valve (FOV) is placed inside the low-pressure plenum 


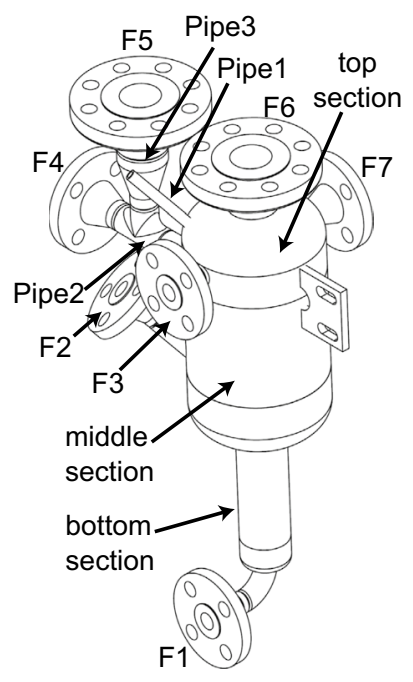

Fig. 4 Drawing of the vapour generator. The connecting flanges are F1 (outlet to extract liquid), F2 (Pt-100 sensor), F3 (return pipe from the LPP), F4 (burst disc), F5 (static pressure transducer), F6 (liquid level meter), F7 (reference tube)

the vapour generator (valves MV-4 and PV-2 closed, see Fig. 2) by means of electrical heaters enveloping the bottom, mid and top sections of the vessel. Preliminary tests highlighted that accumulation of condensed fluid occurred in unheated sections of the vessel, inducing unwanted instabilities in the working fluid temperature when it flows back in the bulk, causing problems with the control system. In order to prevent these phenomena, all the walls of the vapour generator have been heated to limit the condensation of the working fluid.

Most of the thermal energy is supplied to the vapour generator by a $1.5-\mathrm{kW}$ ceramic band heater covering the bottom section, see Fig. 4, because the lower part of the vapour generator is always in contact with liquid. This ensures a high heat transfer coefficient between the heater and the liquid fluid inside the vessel and helps avoiding hot spots that could trigger thermal decomposition of the fluid. A 2-mm graphite layer inserted between the band heater and the metal wall solved initial problems due to imperfect thermal contact between the band heater and the wall, ensuring an even surface temperature distribution. The main section of the vessel is heated by a $2.8-\mathrm{kW}$ ceramic band heater, also in combination with a $2-\mathrm{mm}$ graphite layer. At the top of the vessel, it was impossible to place a band heater due to the presence of numerous flanges. Instead, a 6-m-long $1 \mathrm{~kW}$ Joule dissipation heating wire is used. A second heating wire of the same type is wrapped around pipe 2 and 3, see Fig. 4, and a third one around pipe 4, see Fig. 2, in combination with a 2-mm graphite layer. Heat transfer from the vapour generator to the environment is limited by a layer of minimum $50-\mathrm{mm}$ rockwool insulation. Once the desired pressure is attained in the vapour generator, the manually operated valve MV-4 is opened and vapour flows through pipe 4 into the reference tube (RT) and CT, see Fig. 2.

The purpose of the RT is to finely control the degree of superheating of the vapour and to provide a reference for the thermal control of the CT, as further explained in Sect. 2.2. The RT is a 500-mm-long tube with an internal diameter of $40 \mathrm{~mm}$ and $15-\mathrm{mm}$-thick walls. The thickness of the walls enhances an even distribution of the thermal flux, which is needed to obtain a uniform temperature and to avoid hot spots. The thermal energy is supplied by two heating jackets placed around the tube, which includes a $25-\mathrm{mm}$ glass silk insulation layer. A $335 \mathrm{~W}$ version is placed around the RT and a $180 \mathrm{~W}$ version around the flange of the RT. The heating jacket is chosen because it is designed to provide an even temperature distribution. Around pipe 6, see Fig. 2, a 2.1-m-long $370 \mathrm{~W}$ Joule dissipation heating wire is wrapped with a 2-mm graphite layer placed underneath.

The geometry of the CT and of the RT is identical, except for their length. The CT is composed of six pipe segments, each $1520 \mathrm{~mm}$ long. The pipe segments feature a male-to-female connection, such that the segments overlap for $20 \mathrm{~mm}$, see Fig. 2. The male side has a groove accommodating a red copper seal. A coupling holds the two segments together. This construction allows for satisfactory sealing both in case the inner volume is at superatmospheric pressure or at subatmospheric conditions and for accurate alignment. The $\mathrm{CT}$ assembly measures $9 \mathrm{~m}$ in total and is placed on a sliding support to allow for its thermal expansion when at high temperature, which is $55 \mathrm{~mm}$ at $400{ }^{\circ} \mathrm{C}$. Each pipe segment has been machined from a metal block to ensure maximum straightness and is electrolytically polished on its inner surface to a roughness of $0.05 \mu \mathrm{m}$. Each segment is fitted with a $950 \mathrm{~W}$ glass silk heating jacket, which includes a 25 -mm-thick insulation layer. The couplings between the elements are fitted with two $0.5-\mathrm{m}$ 180-W Joule heating electric wires, covered by a $25-\mathrm{mm}$ glass silk insulation layer. Immersion of temperature sensors in the CT would inherently disturb the flow field of interest. Instead, the outside wall temperature is measured both in the RT and CT. Due to the geometric equivalence of the reference tube and the charge tube, the total length being the only exception, imposing the same temperature on the charge tube walls as on the RT results in the same fluid temperature inside the tube.

The end of the CT is closed off by a FOV, arguably the most complex mechanical part of the set-up, see Fig. 5. This custom-designed valve is able to operate at high temperatures without lubrication to avoid contamination of the working fluid. The valve can be operated remotely, keeping the entire facility hermetically sealed for multiple 


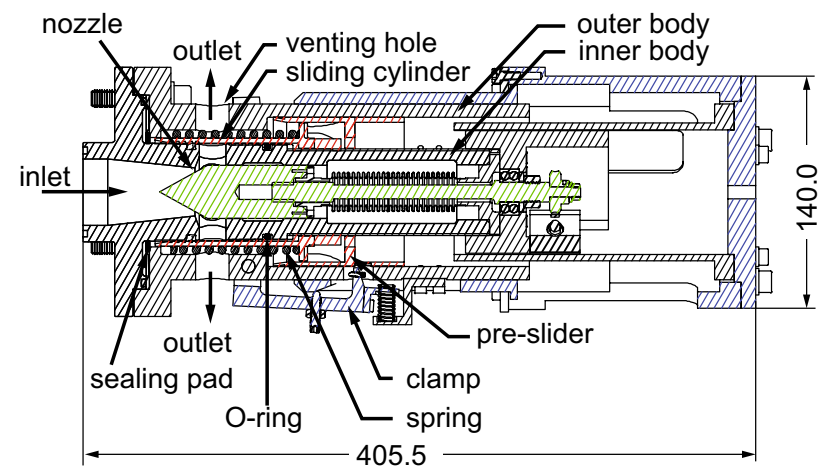

Fig. 5 Drawing of the cross section of the fast-opening valve. The actuation system (in blue) slides slowly to the right side, which pushes the clamps outward. Once the clamps release the pre-slider, the fast-moving components (in red) come into motion because the compressed spring pushes the sliding cylinder and the pre-slider away. After the sliding cylinder is pushed across the venting holes, the fluid is free to flow from the inlet to the outlet. The nozzle insert (in green) can be moved in the longitudinal direction in order to control the throat area

experiments. In the opened position, the working fluid can flow through venting holes present in the inner and outer body in the radial direction. In the closed position, a sliding cylinder is pushed between these bodies, obstructing the flow through the venting holes. The sliding cylinder presses into a Kalrez ${ }^{\circledR, 1}$ sealing pad in order to ensure sealing. On the other side, the sealing is performed by a Kalrez ${ }^{\circledR}$ O-ring with a diameter of $47.22 \mathrm{~mm}$ and a thickness of $3.53 \mathrm{~mm}$ between the sliding cylinder and the inner body. An Inconel steel spring is compressed and three radial clamps engage the pre-slider to prevent the spring from being released. To open the valve, the actuation system moves the clamps in outward direction, allowing the spring to push the sliding cylinder and the pre-slider away, thus leaving the venting holes open. A nozzle insert creates a throat area in order to choke the flow, thus preventing flow disturbances from travelling upstream. For this reason, the throat is located upstream of the sealing, as opposite to solutions that are typical in Ludwieg tubes (Schrijer and Bannink 2010; Knauss et al. 1999). It can be moved remotely in the longitudinal direction to change the throat area section between approximately 420 and $600 \mathrm{~mm}^{2}$, and this allows to modulate the strength of the rarefaction waves.

The FOV is contained in the low-pressure plenum (LPP), a 113-1 cylindrically shaped vessel, see Fig. 2. The LPP has an outer diameter of $406.4 \mathrm{~mm}$ and $9.53-\mathrm{mm}$ thick walls. The electric motor triggering the FOV and the manual nozzle positioning gear are mounted on the

\footnotetext{
$\overline{{ }^{1} \mathrm{Kalrez}^{\circledR} \text { is a registered trademark of the DuPont }}{ }^{\mathrm{TM}}$ Company.
}

LPP with feedthrough shaft connections sealed with copper gaskets. The thermal input to the vessel is supplied by four heating jackets with a nominal power of 1450 , 425, 960 and $490 \mathrm{~W}$, respectively, each featuring also a 25-mm glass silk insulation layer.

The pneumatic globe valve PV-1, see Fig. 2, connects the LPP to a finned air-cooled condenser equipped with a $60 \mathrm{~W}$ fan. The condensed liquid flows from the bottom of the condenser into the 24.8-mm inner diameter inclined flow return pipe that has 1-mm-thick walls, where the liquid is collected. This pipe is connected through the pneumatic globe valve PV-2 at flange F3, see Fig. 4, such that the fluid can flow into the vapour generator.

\subsection{Instruments, data acquisition and control}

Two separate subsystems are implemented and connected to a personal computer for data visualization, monitoring and post-processing: one is specifically for the acquisition of data related to experiments, while the other operates to monitor and control the fluid conditions in the facility.

The monitoring and control is performed by two programmable automation controllers (PAC). The code related to the data acquisition functionality and the code related to the control algorithms are implemented into the FAST Manager program, an in-house NI LabVIEW ${ }^{\mathrm{TM}}$ program partly running on the PACs (for the critical control functions) and partly on the personal computer.

The thermal energy supply to the various sections of the facility is regulated by digital PID controllers, and the voltage supply to the heating elements is modulated via thyristors.

In the vapour generator, the static pressure had initially been used as process variable for the main heater at the bottom section, measured by a pressure transmitter connected at flange F5 in Fig. 4 with a specified accuracy of $0.1 \%$ of the full range of 10 bar. Because the operating pressure range of the vapour generator spans from very far from the critical point to close-to-critical conditions, the response in pressure to the supplied thermal power changes dramatically, because of the variation in the pressure derivative with respect to temperature at constant specific volume $(\partial P / \partial T)_{v}$. Consequently, the PID parameters should also be adapted depending on the operated pressure. This is avoided by converting the pressure into a saturation temperature with the help of in-house software (Colonna et al. 2012) implementing suitable fluid thermodynamic property models (see e.g. Colonna et al. 2008b). Now the same PID parameters can be used throughout the entire operating range, because the change in specific heat capacity is sufficiently moderate. At the same time, the fast response of the pressure transmitter is still exploited for the control. The 
fluid saturation temperature is compared by a direct measurement of the liquid temperature as sensed by a Pt-100 sensor mounted on flange F2, see Fig 5. The accuracy of all the Pt-100 sensors used in the set-up has been evaluated as $0.12{ }^{\circ} \mathrm{C}$ resulting from an in-house calibration process.

The other heaters on the vapour generator are regulated, each based on the temperature difference as measured by a 1-mm-thick K-type thermocouple and the fluid temperature. Additionally, five thermocouples are placed for monitoring purposes to detect hot spots and to monitor whether an even temperature distribution is obtained. All thermocouples are placed underneath the heater between the wall and the graphite layer. The liquid level in the vessel is monitored by a radar level meter mounted at flange F6 (Fig. 4).

The heat supply to the RT is regulated by a PID controller using as process variable, the difference in temperature between the liquid in the vapour generator and the vapour in the RT, both measured with a Pt-100. The temperature in the $\mathrm{CT}$ is monitored with an identical instrument.

The fluid temperature inside the CT is controlled by imposing the same wall temperature on the $\mathrm{CT}$ as on the RT, which are geometrically equivalent, except for the length. The process variables are the differences in wall temperature between the RT and the individual CT pipe segments and couplings. Instead of measuring the temperature of the two thermocouples and calculating the difference in temperature, here the two thermocouples are connected to each other and the voltage difference is directly measured to reduce the measurement error. The wall temperature is measured in a groove in the RT and in each $\mathrm{CT}$ element or CT coupling using a 0.5-mm-diameter thermocouple of $1 \mathrm{~m}$ length, which is bond to the tube with a silver-filled conductive ceramic adhesive to ensure the correct measurement of the wall temperature.

Less accuracy is required for the thermal control of the LPP, because its only purpose is to limit temperature gradients in the charge tube by reducing heat conduction. Therefore only a single controller is implemented in order to regulate the power supply to all the heaters on the LPP. The process variable is the measured temperature with a Pt- 100 .

The signals from Pt-100s TE1.0, TE2.0 and TE4.0 (Fig. 2) go through a transmitter to convert the signal into a linear 4-20 mA one. Consequently, these signals, together with the other 4-20 mA signals from liquid level meter LIT1 and from the static pressure transducers PIT5-PIT7, are converted to a voltage signal using a $249 \Omega$ resistance and connected to the PAC through a 16-bit analog input module. The thermocouple signals are connected using a 16-bit input module specific for these sensors.

Four dynamic pressure measurement stations PT1-PT4 are created by flush mounting a high-temperature fully active four-arm Wheatstone bridge pressure transducer that measures the pressure with an accuracy of $0.5 \%$ of the full scale of 21 bar along the CT at a distance of 4, 4.3, 8.4 and $8.7 \mathrm{~m}$ from the FOV, respectively, see Fig. 2. The signals are scaled after each experiment with the more accurate value of the pressure before and after the experiment, measured by the static pressure transducers. The sensors are placed in pairs to give the possibility to have a time-offlight measurement at two different locations in the tube. The $0-100 \mathrm{mV}$ signals of the dynamic pressure transducers are amplified with a custom-built amplifier with a gain of 100 and connected to a 16-bit data acquisition card that digitizes the signal in a simultaneous manner at a frequency of $250 \mathrm{kHz}$. A rise time of the pressure signals of $30 \mu \mathrm{s}$ has been measured using a shock test. The Pt-100 in the CT (TE3.0) is connected to a separate 16-bit data acquisition card.

\section{Facility characterization using incondensable gases}

Avoiding contamination of the working fluid with air and/ or moisture is of utmost importance. A comprehensive series of tests have been conducted up to temperatures of $350{ }^{\circ} \mathrm{C}$, for a duration of $72 \mathrm{~h}$ each, to evaluate the leak rate of the facility. The test results allowed to assess that the temperature has a negligible influence on the sealing properties of the equipment. The tightness of the FAST setup has been characterized in terms of the average leak rate $\mathrm{LR}=\Delta p V \Delta t^{-1}$ (Moore 1998), where $V$ is the volume of the whole set-up (i.e. $0.143 \mathrm{~m}^{3}$ ), and $\Delta p$ is the pressure drop/rise measured after a time interval $\Delta t$ (i.e. 3 days for all the tests). For subatmospheric pressure levels, i.e. $p \lesssim 3$ mbar abs., LR values lower than $5 \times 10^{-4} \mathrm{mbar}^{1 \mathrm{~s}^{-1}}$ were obtained (corresponding to air leaking into the system). For superatmospheric pressure levels, i.e. $p \gtrsim 6$ bar abs., the tests were conducted filling the set-up with helium, and the measured LR values were lower than $5 \times 10^{-2}{\text { mbar } 1 \mathrm{~s}^{-1}}^{-1}$ (helium to the ambient).

A series of rarefaction wave experiments are performed in order to characterize the opening time of the FOV. The expansion is obtained by pressurizing the $\mathrm{CT}$ and by keeping the LPP at a lower pressure. After the FOV opens, a rarefaction travels into the $\mathrm{CT}$, decreasing the pressure from stagnant state A to state $\mathrm{B}$, and starting a flow through the nozzle into the LPP, see Fig. 1. In the throat of the nozzle, sonic conditions are attained.

The experiment is illustrated in a position-time diagram in Fig. 6, in which exemplary characteristic curves are displayed. If the opening of the valve were instantaneous, all characteristics would emerge from the origin of the chart. In a straight pipe without any area changes, the characteristics are straight lines as shown in Fig. 7a. In the case of the actual valve opening, featuring a finite opening time, the 


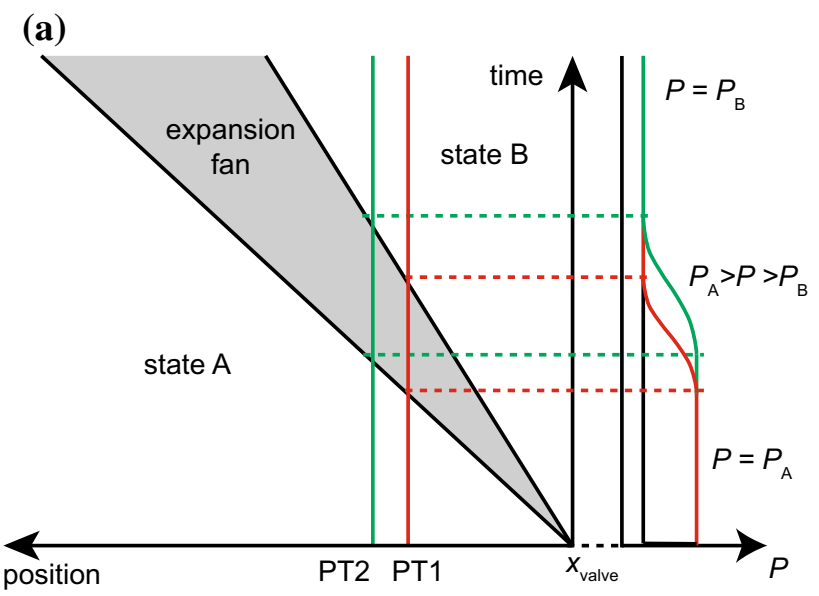

(b)

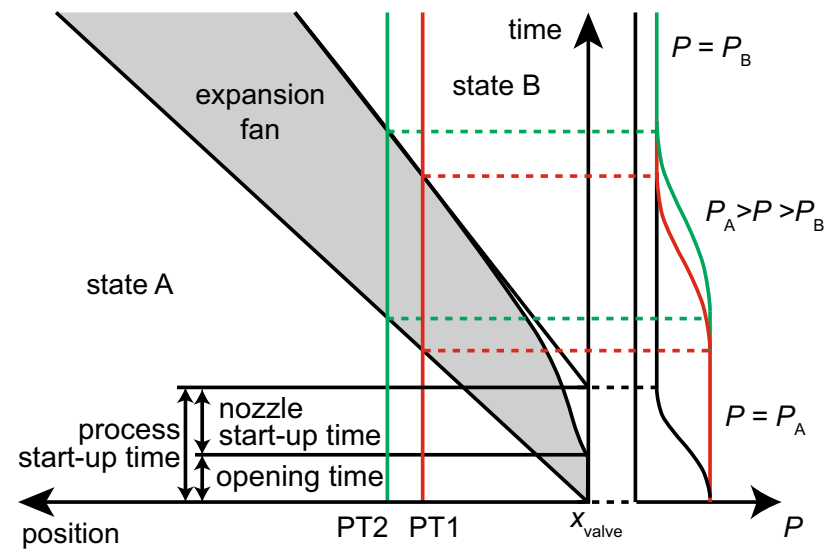

Fig. 6 Qualitative position-time diagrams referred to classical expansion fans, with corresponding pressure diagrams at measurement stations PT1 and PT2. In the ideal case (instantaneous valve opening) and in the absence of a nozzle, all characteristics emerge from a single point and the pressure drop is instantaneous. In the actual case, due to the finite FOV opening time, the pressure drop is not instantaneous and consequently, the limiting characteristic starts at a later time instance as the first characteristic. All characteristics except the first one are curved in the nozzle area, because of the flow acceleration in this section, which decreases the wave propagation speed. a Ideal case with instantaneous valve opening and no nozzle, $\mathbf{b}$ real case with non-instantaneous valve opening and nozzle

limiting characteristic emerges from the valve position at a later time instance, see Fig. 7b. Moreover, since the seal of the FOV is downstream of the nozzle, the rarefaction propagates through the nozzle before entering the CT. The flow is accelerated in the nozzle, resulting in a significantly lower wave propagation speed than in the rest of the tube, and consequently, the characteristics are curved in this section (Cagliostro and Johnson 1971).

Figure 7 shows the pressure measurements of a rarefaction experiment (no. 25 from Table 1) in nitrogen with an initial pressure of 4.01 bar in the CT and vacuum in the LPP. From these data, the FOV opening sequence can be

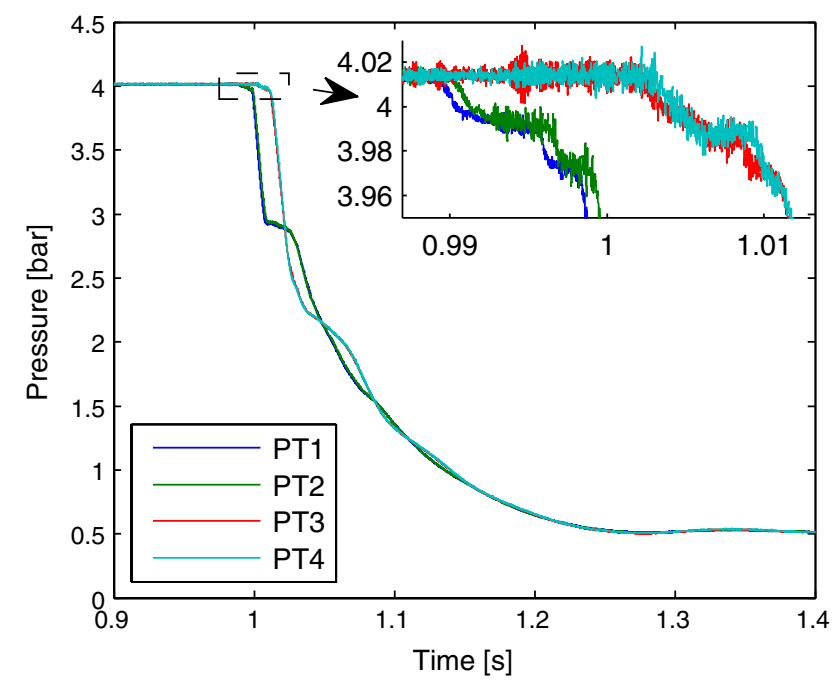

Fig. 7 Pressure signals of experiment 25 measured by transducers PT1 to PT4. The valve opening results in a pressure drop from 3.97 bar to 2.88 bar, which is preceded by two other small pressure drops. These are attributed to an initial leakage flow that starts as soon as the FOV sliding cylinder detaches from the seal

inferred: as soon as the clamps are released, see Fig. 5, the spring pushes the slider away, and a small opening is created as the slider leaves the seal, choking the flow close to this position. This causes a small pressure drop of approximately 20 mbar in the signal. As the slider moves further, the position of minimum cross section changes to a section between the slider and the inner body, which is slightly larger than the initial choked section. This is revealed by a second small drop in the pressure of approximately 20 mbar. Only when the slider passes over the venting hole of the inner body, the designated nozzle gets choked. This corresponds to the large pressure drop in the pressure signal down to 2.88 bar.

The local wave propagation speed equals $w=c-u$ for left running waves, in which $c$ and $u$ are the local speed of sound and local flow velocity, respectively. An experimental value of the wave propagation speed is obtained with the time-of-flight method. First, the relevant part of the pressure signal is selected as follows: the beginning of the wave at $t=t_{\text {start }}$ is formally identified as the time when the pressure departs from the initial value $P_{\mathrm{CT}}$ by more than 15 mbar. The threshold is chosen such that it exceeds the noise level. The end of the unperturbed portion of the signal is chosen when the head of the wave is expected to reach the end of the CT at $t=t_{\text {end }}$. The estimate is performed by using the speed of sound from the accurate nitrogen thermodynamic model, which is equal to the ideal gas model in this regime; therefore, $t_{\mathrm{end}}=t_{\mathrm{start}}+\Delta x / c_{\text {model }}$, where $\Delta x$ is the distance between the sensor location and the end of the CT. The relevant portion of the signal $\Delta P_{\text {rel }}$ spans 
from $P_{\text {start }}$ to $P_{\text {end }}$. This span is divided into intervals of $0.64 \mathrm{mbar}$, equal to two times the resolution of the pressure signal. The time-of-flight method is applied to corresponding subintervals from different sensors to compute the local wave propagation speed.

A theoretical curve is obtained by evaluating the speed of sound and flow velocity as a function of the pressure drop. The speed of sound is determined using the thermodynamic model which corresponds to the ideal gas model in this regime and assuming an isentropic expansion. The local flow velocity is obtained by assessing the Riemann invariant in the undisturbed state, which is constant in this simple wave (Thompson 1988). Figure 8 shows for the example of experiment 25 the chart that can be obtained to this end. Wave speed values as a function of the measured pressure drop are shown together with the theoretical curve and two fitting curves: a linear fit and a shift of the theoretical curve with the average deviation from the experimental curve.

The fitting curves give an estimate of the speed of sound in the undisturbed state, as the wave propagation speed tends to the speed of sound as $\Delta P \rightarrow 0$. To eliminate inaccuracies introduced by the FOV, only the first $25 \%$ of the relevant pressure span is taken, while keeping a minimum of 40 mbar, such that $\Delta P_{\max }=\max \left(0.25 P_{\text {rel }}, 0.04\right.$ bar $)$. The resulting speed of sound $c_{\text {fit }}$ and $c_{\text {offset }}$ is reported in Table 1. For air, $\mathrm{CO}_{2}$ and $\mathrm{N}_{2}$, both methods deliver an accurate estimate of the speed of sound, compared to the value

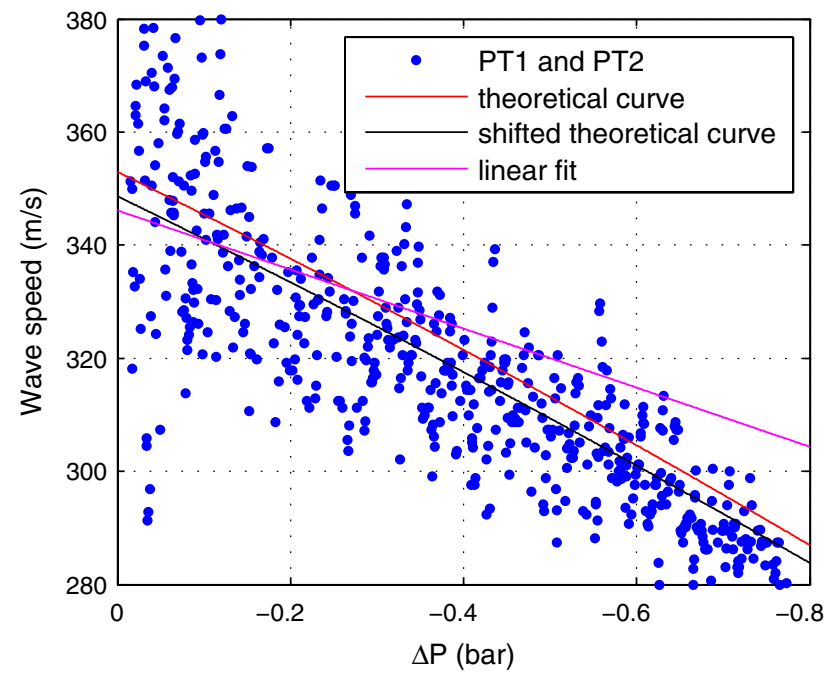

Fig. 8 Wave Speed in $\mathrm{N}_{2}$ as a function of the pressure drop. The blue dots are the values obtained from pressure signals by applying the time-of-flight method. The red line is the theoretical curve as obtained with a reference thermodynamic model. The black line is a fit of the values obtained with the experiments using an offset of the theoretical curve. The magenta line is a linear fit of the data. The shifted theoretical curve and the linear fit are made based on $25 \%$ of $\Delta P_{\text {rel }}=-0.2$ bar obtained from the reference thermodynamic model. The only exception is the linear fit for experiment 23 . The average error and standard deviation with respect to the model are $2.1 \% \pm 1.6 \%$ and $1.1 \% \pm 1.1 \%$ for the linear fit and the theoretical offset, respectively. For helium, the average error and standard deviation with respect to the model are $5.1 \% \pm 4.6 \%$ and $4.0 \% \pm 3.1 \%$ for the linear fit and the shifted theoretical curve, respectively. The reason for the larger error is due to the finite process start-up time of the FOV in combination with the higher speed of sound of helium resulting in a very small usable portion of the signal.

The process start-up time of the FOV is determined by reconstructing the pressure signal at the valve location from the original pressure signals and by subtracting, for each data point, the propagation time of a wave from the valve location to the sensor location at the local wave speed $t_{\text {rec }}=t-w \Delta x$, see Fig. 7b. In the reconstructed signal, the time duration of the pressure drop corresponds to the process start-up time.

Figure 9 shows the resulting signals at the valve location of all four sensors for experiment 25, see Table 1 . Since the signals are mapped to the same location, i.e. the valve location, the initial portion of all signals coincides. Below a pressure level of 3.7 bar, signals from sensor PT3 and PT4 deviate from the other two signals. Such deviation can be explained by the reflection of the rarefaction waves once they reach the end of the tube, which creates a non-simple region (Thompson 1988). The local flow velocity is then

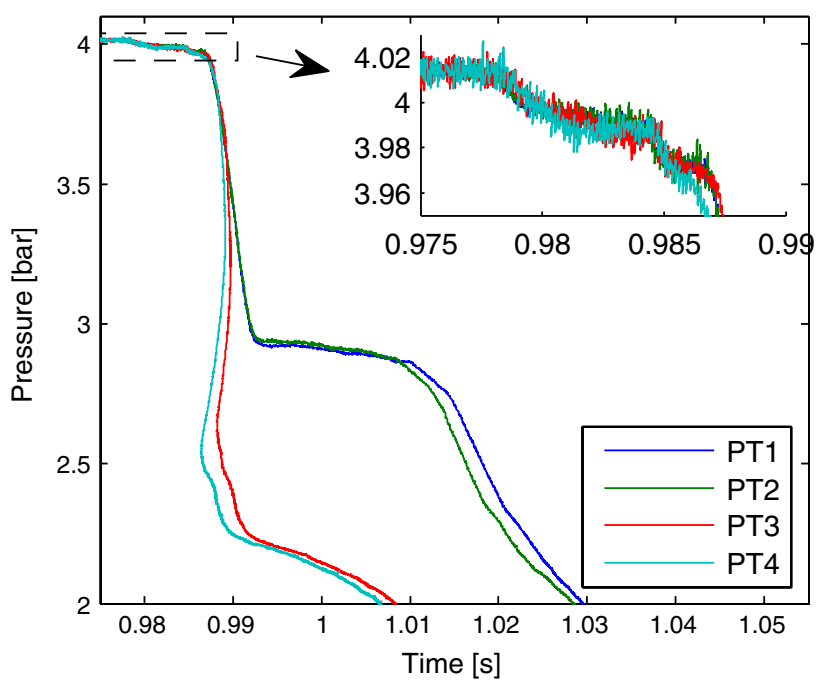

Fig. 9 Section of the signals recorded by pressure sensor PT1 to PT4 during an expansion in nitrogen mapped to the valve position. All signals initially overlap, demonstrating that the mapping procedure is correct. The PT3 and PT4 signals start to deviate from the other two signals once the rarefaction is reflected at the end of the tube, thus creating a non-simple region 
Table 1 Results from rarefaction measurements

\begin{tabular}{|c|c|c|c|c|c|c|c|c|c|c|c|}
\hline No. & Fluid & $\begin{array}{l}P_{\mathrm{CT}} \\
\text { (bar) }\end{array}$ & $\begin{array}{l}T_{\mathrm{CT}} \\
\left({ }^{\circ} \mathrm{C}\right)\end{array}$ & $\begin{array}{l}\Gamma \\
(-)\end{array}$ & $\begin{array}{l}\Delta P_{\text {wave }} \\
\text { (bar) }\end{array}$ & $\begin{array}{l}A_{\text {geom }} \\
\left(\mathrm{mm}^{2}\right)\end{array}$ & $\begin{array}{l}A_{\text {eff }} \\
\left(\mathrm{mm}^{2}\right)\end{array}$ & $\begin{array}{l}\Delta t \\
(\mathrm{~ms})\end{array}$ & $\begin{array}{l}c_{\text {model }} \\
(\mathrm{m} / \mathrm{s})\end{array}$ & $\begin{array}{l}c_{\text {fit }} \\
(\mathrm{m} / \mathrm{s})\end{array}$ & $\begin{array}{l}c_{\text {offset }} \\
(\mathrm{m} / \mathrm{s})\end{array}$ \\
\hline 1 & $\mathrm{He}$ & 6.01 & 17.3 & 1.34 & 1.74 & 460 & 459 & 3.9 & 1006 & 998 & 964 \\
\hline 2 & $\mathrm{He}$ & 4.90 & 22.7 & 1.34 & 0.28 & 70 & 79 & 2.7 & 1014 & 1014 & 1028 \\
\hline 3 & $\mathrm{He}$ & 6.09 & 48.9 & 1.34 & 1.69 & 460 & 438 & 3.7 & 1059 & 1005 & 1072 \\
\hline 4 & $\mathrm{He}$ & 6.50 & 50.0 & 1.34 & 0.32 & 70 & 67 & 2.5 & 1060 & 1121 & 955 \\
\hline 5 & $\mathrm{He}$ & 6.66 & 99.2 & 1.34 & 2.01 & 460 & 483 & 3.6 & 1138 & 1274 & 1169 \\
\hline 6 & $\mathrm{He}$ & 6.02 & 149.4 & 1.34 & 1.84 & 460 & 489 & 4.1 & 1212 & 1225 & 1182 \\
\hline 7 & $\mathrm{He}$ & 7.29 & 269.4 & 1.34 & 0.33 & 70 & 62 & 2.1 & 1373 & 1547 & 1348 \\
\hline 8 & $\mathrm{He}$ & 8.47 & 269.9 & 1.34 & 0.44 & 70 & 72 & 3.2 & 1374 & 1424 & 1483 \\
\hline 9 & Air & 6.31 & 17.6 & 1.21 & 1.62 & 460 & 456 & 4.2 & 343 & 341 & 341 \\
\hline 10 & Air & 4.08 & 18.0 & 1.20 & 0.97 & 460 & 418 & 4.2 & 343 & 338 & 339 \\
\hline 11 & Air & 7.01 & 19.1 & 1.21 & 1.81 & 460 & 459 & 4.2 & 344 & 338 & 339 \\
\hline 12 & Air & 4.86 & 20.0 & 1.21 & 0.21 & 70 & 67 & 2.3 & 344 & 359 & 350 \\
\hline 13 & Air & 5.39 & 20.0 & 1.21 & 0.23 & 70 & 68 & 2.3 & 344 & 344 & 353 \\
\hline 14 & Air & 7.13 & 22.0 & 1.21 & 0.30 & 70 & 67 & 2.2 & 345 & 338 & 347 \\
\hline 15 & Air & 6.15 & 49.1 & 1.21 & 1.62 & 460 & 468 & 4.2 & 361 & 353 & 356 \\
\hline 16 & Air & 6.98 & 95.0 & 1.20 & 0.27 & 70 & 61 & 2.2 & 386 & 375 & 388 \\
\hline 17 & Air & 6.80 & 99.5 & 1.20 & 1.76 & 460 & 461 & 4.0 & 388 & 390 & 384 \\
\hline 18 & $\mathrm{CO}_{2}$ & 5.99 & 18.0 & 1.13 & 1.50 & 460 & 471 & 4.9 & 262 & 257 & 259 \\
\hline 19 & $\mathrm{CO}_{2}$ & 6.42 & 50.0 & 1.12 & 1.59 & 460 & 465 & 4.6 & 275 & 274 & 273 \\
\hline 20 & $\mathrm{CO}_{2}$ & 6.64 & 100.3 & 1.12 & 1.64 & 460 & 464 & 4.1 & 296 & 294 & 293 \\
\hline 21 & $\mathrm{CO}_{2}$ & 6.13 & 149.7 & 1.11 & 1.50 & 460 & 459 & 4.4 & 314 & 321 & 315 \\
\hline 22 & $\mathrm{~N}_{2}$ & 1.09 & 25.3 & 1.20 & 0.35 & 600 & 576 & 4.3 & 352 & 337 & 338 \\
\hline 23 & $\mathrm{~N}_{2}$ & 1.12 & 25.4 & 1.20 & 0.33 & 600 & 531 & 4.4 & 352 & 379 & 349 \\
\hline 24 & $\mathrm{~N}_{2}$ & 4.00 & 25.7 & 1.20 & 1.00 & 460 & 443 & 4.1 & 353 & 360 & 353 \\
\hline 25 & $\mathrm{~N}_{2}$ & 4.01 & 25.8 & 1.20 & 1.08 & 460 & 481 & 4.2 & 353 & 346 & 349 \\
\hline 26 & $\mathrm{D}_{6}$ & 1.26 & 264.2 & 0.86 & 0.09 & 600 & 169 & 7.7 & 89.8 & 89.5 & 88.4 \\
\hline 27 & $\mathrm{D}_{6}$ & 1.26 & 293.7 & 0.91 & 0.15 & 600 & 278 & 6.6 & 94.3 & 94.0 & 94.1 \\
\hline 28 & $\mathrm{D}_{6}$ & 1.27 & 298.0 & 0.91 & 0.18 & 460 & 328 & 7.5 & 94.8 & 96.9 & 93.9 \\
\hline 29 & $\mathrm{D}_{6}$ & 1.27 & 300.1 & 0.92 & 0.14 & 600 & 254 & 5.6 & 95.1 & 98.3 & 94.0 \\
\hline 30 & $\mathrm{D}_{6}$ & 2.52 & 301.1 & 0.79 & 0.26 & 460 & 254 & 9.0 & 84.6 & 84.9 & 83.5 \\
\hline 31 & $D_{6}$ & 2.38 & 302.3 & 0.81 & 0.21 & 600 & 220 & 8.3 & 86.2 & 83.5 & 82.7 \\
\hline 32 & $\mathrm{D}_{6}$ & 2.53 & 305.1 & 0.79 & 0.29 & 460 & 286 & 8.9 & 85.4 & 84.3 & 83.5 \\
\hline
\end{tabular}

$P_{\mathrm{CT}}$ and $T_{\mathrm{CT}}$ are the measured initial pressure and temperature in the charge tube. $\Gamma$ is the fundamental derivative of gas dynamics as predicted by the reference equation of state for the measured thermodynamic conditions. The $\Delta P_{\text {wave }}$ is the pressure difference measured across the rarefaction. $A_{\text {geom }}$ is the nozzle area as derived from the geometry. $A_{\text {eff }}$ is the effective nozzle area calculated using the measured pressure drop across the expansion. $\Delta t$ is the process start-up time inferred from the mapped signal. $c_{\text {model }}$ is the speed of sound estimated using the most accurate thermodynamic model for the measured conditions (uncertainty unknown). $c_{\text {fit }}$ is the speed of sound as measured by fitting the experimental time-of-flight results using a linear function. $c_{\text {offset }}$ is the speed of sound obtained by shifting the theoretical curve with the average deviation from the experimental results incorrectly evaluated, and it makes PT3 and PT4 unsuited for the determination of the process start-up time.

The process start-up time has been evaluated for several experiments using $\mathrm{He}$, air, $\mathrm{CO}_{2}$ and $\mathrm{N}_{2}$ as working fluids, different pressure levels and nozzle areas, see Table 1 , experiments 1 to 25 . Given the need of comparing opening times at various pressure levels, temperature and fluids, we define the process start-up time as the time duration between $5 \%$ of the pressure drop and $95 \%$ of the pressure drop of the mapped signal has occurred. The boundaries have been chosen such that the signal maximum noise level does not exceed these values. As expected, the value of the throat area in the nozzle affects the measured process startup time.

The effective nozzle area, which also depends on multidimensional and viscous effects, is not easily recovered 
from the FOV geometry, and therefore, it is inferred from the measurements by assuming a steady quasi-one-dimensional isentropic flow from state B through the choked nozzle. The mass flow is evaluated using the known crosssectional area of the CT, the evaluated flow velocity using the Riemann invariant from state $\mathrm{A}$ and the density of state $\mathrm{B}$, which is calculated by using the isentropic relations from state A. With a very small calculated throat area of 61-79 $\mathrm{mm}^{2}$, performed with a special nozzle insert, the measured process start-up time is between 2.1 and $3.2 \mathrm{~ms}$, see Table 1 . Without the nozzle insert, the calculated throat area is between 418 and $576 \mathrm{~mm}^{2}$, and the process startup time is between 3.5 and $4.9 \mathrm{~ms}$ for all experiments with incondensable gases.

\section{Rarefaction waves in siloxane $D_{6}$}

In order to demonstrate the correct operation of the set-up for the functionality it was designed for, i.e. wave measurements in dense vapours of organic fluids, experiments to measure the expansion wave speed in the non-ideal region of $\mathrm{D}_{6}$ (dodecamethylcyclohexasiloxane, $\mathrm{C}_{12} \mathrm{H}_{36} \mathrm{O}_{6} \mathrm{Si}_{6}$ ) are performed. Gaschromatographic analysis confirmed the specification of the supplier that the fluid has a $96 \%$ purity. Experiments at two different saturation levels are conducted with the aim of evaluating how the control system is able to keep the temperature constant and uniform at different temperature levels. The thermodynamic conditions are reported in Table 1 together with the predicted value of $\Gamma$. Figure 10 shows the temperature of the fluid in the vapour generator and in the reference tube during the entire test campaign. Part of the liquid in the vapour generator is flashed once valve MV-4 is opened. The measured temperature fluctuations in the reference tube and charge tube have a period of approximately $2 \mathrm{~h}$ with an amplitude of up to $3{ }^{\circ} \mathrm{C}$.

Pressure recordings of experiment 28 are displayed in Fig. 11. The process start-up time is determined by applying the same mapping procedure as illustrated in Sect. 3 and is found to be between 5.6 and $9.0 \mathrm{~ms}$ in all experimental runs. The nozzle area is varied between 460 and $600 \mathrm{~mm}^{2}$ based on the geometry. Calculations using the resulting pressure drop from the experiments result in a significantly lower nozzle area, see Table 1 , which may indicate that there is accumulation of condensed fluid in the unheated FOV.

In order to verify that the measurements are coherent with the measured operating conditions, the speed of sound has been computed from the experimental data and compared with estimations obtained with the best available, though possibly still inaccurate, thermodynamic model for pure $\mathrm{D}_{6}$ (Colonna et al. 2008b). Using the estimated

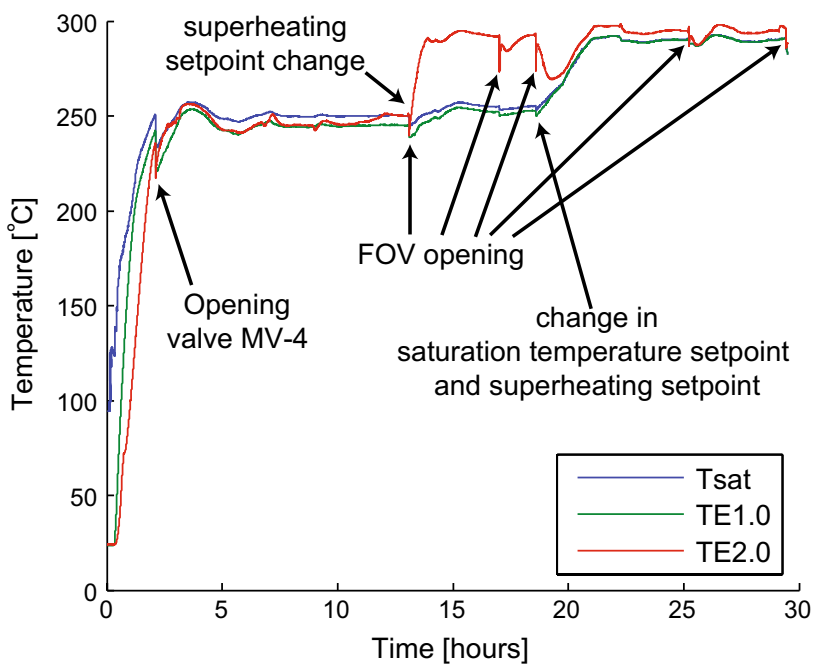

Fig. 10 Temperature recordings during the experimental campaign on siloxane $\mathrm{D}_{6} . \mathrm{T}_{\text {sat }}$ is obtained from the measured pressure in the vapour generator by means of the suitable thermodynamic model from Colonna et al. (2008b). TE1.0 is the temperature measured by the Pt-100 sensor in the vapour generator. TE2.0 is the temperature measured by the Pt-100 sensor in the reference tube

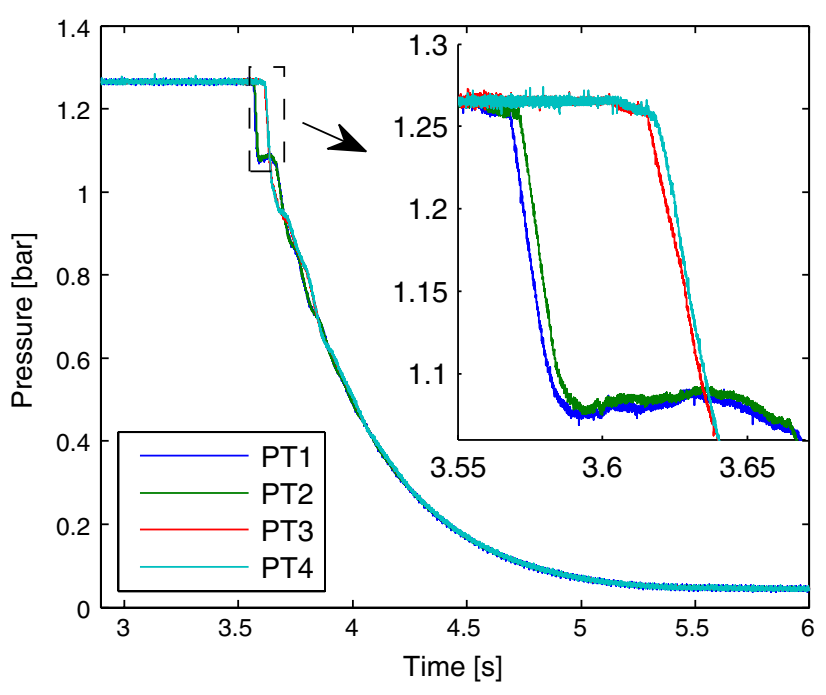

Fig. 11 Pressure recordings during a $\mathrm{D}_{6}$ experiment. The conditions in the CT are 1.27 bar and $298^{\circ} \mathrm{C}$. PT1 to PT4 are the pressure recordings of the sensor closest to the FOV to furthest away from the FOV, respectively

sound speed, the theoretical curve is constructed in the same manner as for the other gases, except that the ideal gas model cannot be used in this case, and consequently, the Riemann invariant cannot be integrated analytically. The local flow velocity is instead evaluated by integrating the Riemann invariant numerically. Results obtained from experiment 28 are displayed in Fig. 12. The experimental 


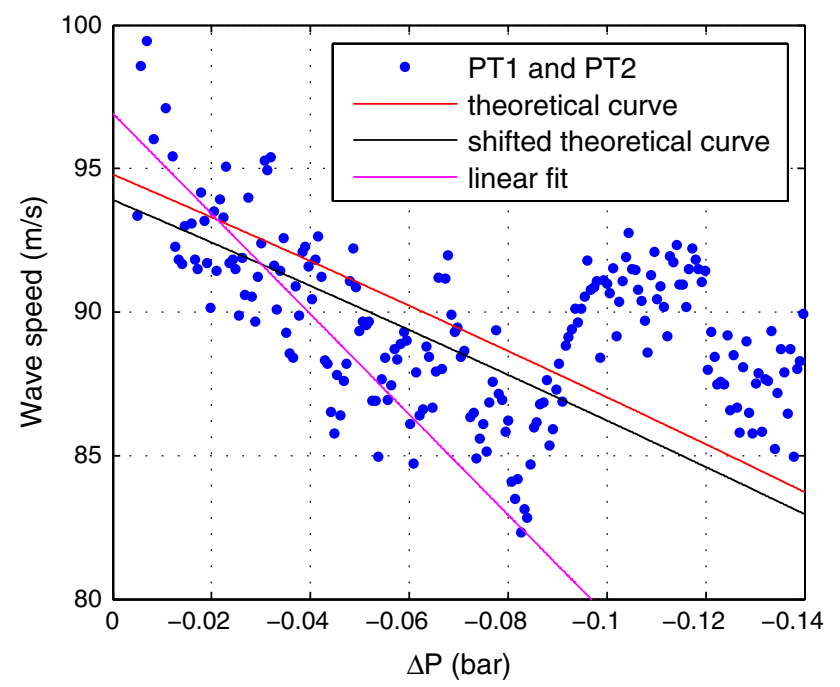

Fig. 12 Wave speed in $D_{6}$ siloxane as a function of the pressure drop. The blue dots are the experimental results obtained with the time-of-flight measurements. The red line is the theoretical curve as calculated by the thermodynamic model from Colonna et al. (2008b). The black line is a fit of the experimental data using an offset of the theoretical curve. The magenta line is a linear fit of the data

local wave propagation speed is evaluated using the timeof-flight method as described in Sect. 3 for sensors PT1/ PT2. The measurements of sensors PT3/PT4 are influenced by the reflection of the rarefaction from the CT end wall. The wave speed calculated from experimental data for this experiment is within $8 \%$ of the value predicted by the thermodynamic model, except for a pressure drop lower than 12 mbar. This is possibly because the pressure gradient is still very low for that portion of the signal, making the influence of noise more significant. By fitting the experimental data in the same manner as illustrated for the case of incondensable gases, i.e. using a linear fit, and by adopting an offset of the theoretical curve, the speed of sound $c_{\mathrm{fit}}$ and $c_{\text {offset }}$ is obtained. The average error and standard deviation with respect to the value predicted by the thermodynamic model are $1.6 \% \pm 1.2 \%$ for the linear fit and $1.6 \%$ $\pm 1.1 \%$ for the theoretical offset. The experimental results are surprisingly close to model predictions, given the fact that, differently from the case of incondensable gas, the $\mathrm{D}_{6}$ thermodynamic model is expected to be rather inaccurate for states in the close proximity of the saturation curve. Moreover, the model is valid for pure $\mathrm{D}_{6}$, while $4 \%$ of the working fluid is composed of other components.

\section{Conclusion and future work}

A novel Ludwieg tube-type facility has been commissioned at Delft University of Technology to perform measurements on waves propagating in NICFD flows. The pressure and temperature of the working fluid can be regulated independently from each other, such that any thermodynamic state can be achieved within the limits of the measurement system of 21 bar and $400{ }^{\circ} \mathrm{C}$. A procedure to estimate the process start-up time of the FOV has been devised, and estimated values range from 2.1 to $9.0 \mathrm{~ms}$. A method to estimate the speed of sound from wave propagation measurements is presented and found to agree within $2.1 \%$ of the predicted value for a variety of incondensable gases. Preliminary experiments on rarefaction waves in the non-ideal regime of siloxane $\mathrm{D}_{6}$ at temperatures up to $300{ }^{\circ} \mathrm{C}$ have been successfully performed, providing values of the wave speed that are within $8 \%$ and speed of sound within $1.6 \%$ of the predictions of a state-of-the-art thermodynamic model. Estimates of the speed of sound calculated with this thermodynamic model are affected by $30 \%$ uncertainty in the region of interest.

More experiments are planned at higher temperatures and closer to the saturation line, where minimum values of $\Gamma$ are predicted. For these measurements, the regulation of the temperature in the charge tube should be refined and the fluctuations are considerably reduced if compared to the current $3{ }^{\circ} \mathrm{C}$, by means of optimization of the control logic, in order to avoid condensation.

Open Access This article is distributed under the terms of the Creative Commons Attribution 4.0 International License (http://creativecommons.org/licenses/by/4.0/), which permits unrestricted use, distribution, and reproduction in any medium, provided you give appropriate credit to the original author(s) and the source, provide a link to the Creative Commons license, and indicate if changes were made.

\section{References}

Brown B, Argrow B (2000) Application of Bethe-Zel'dovich-Thompson fluids in organic Rankine cycle engines. J Propul Power 16(6):1118-1124

Cagliostro D, Johnson J III (1971) Starting phenomena in a supersonic tube wind tunnel. AIAA J 9(1):101-105

Calderazzi L, Colonna P (1997) Thermal stability of R-134a, R-141b, R-13I1, R-7146, R-125 associated with stainless steel as a containing material. Int J Refrig 20(6):381-389

Colonna P, Guardone A, Nannan N, Zamfirescu C (2008a) Design of the dense gas flexible asymmetric shock tube. J Fluids Eng T ASME 130(3):034501

Colonna P, Nannan NR, Guardone A (2008b) Multiparameter equations of state for siloxanes: $\left[\left(\mathrm{CH}_{3}\right)_{3}-\mathrm{Si}-\mathrm{O}_{1 / 2}\right]_{2}-$ $\left.\left(\mathrm{CH}_{3}\right)_{2}\right]_{i=1, \ldots, 3}$. Fluid Phase Equilib 263(2):115-130

Colonna P, van der Stelt TP, Guardone A (2012) FluidProp (Version 3.0): a program for the estimation of thermophysical properties of fluids. http://www.asimptote.nl/software/fluidprop

Colonna P, Casati E, Trapp C, Mathijssen T, Larjola J, Turunen-Saaresti T, Uusitalo A (2015) Organic Rankine cycle power systems: from the concept to current technology, applications, and an outlook to the future. J Eng Gas Turbines Power T ASME 137:1-19 
Conboy T, Wright S, Pasch J, Fleming D, Rochau G, Fuller R (2012) Performance characteristics of an operating supercritical $\mathrm{CO}_{2}$ Brayton cycle. J Eng Gas Turbines Power 134(11):1-12

Cramer MS (1989) Shock splitting in single-phase gases. J Fluid Mech 199:281-296

Dvornic PR (2004) High temperature stability of polysiloxanes. Silicon compounds: silanes and silicones. Gelest Catalog, Gelest Inc, Morrisville, pp 419-432

Fergason SH, Guardone A, Argrow BM (2003) Construction and validation of a dense gas shock tube. J Thermophys Heat Transf 17(3):326-333

Guardone A (2007) Three-dimensional shock tube flows of dense gases. J Fluid Mech 583:423-442

Knauss H, Riedel R, Wagner S (1999) The shock wind tunnel of stuttgart university - a facility for testing hypersonic vehicles. In: 9th international space planes and hypersonic systems and technologies conference, AIAA 99-4959

Maerefat M, Fujikawa S, Akamatsu T, Goto T, Mizutani T (1989) An experimental study of non-equilibrium vapour condensation in a shock-tube. Exp Fluids 7(8):513-520

Moore PO, Jackson CN, Sherlock CN (eds) (1998) Nondestructive testing handbook, vol 1. Leak testing, 3rd edn. The American Society for Nondestructive Testing

Nannan N, Colonna P, Tracy C, Rowley R, Hurly J (2007) Idealgas heat capacities of dimethylsiloxanes from speed-of-sound measurements and ab initio calculations. Fluid Phase Equilib 257(1):102-113

Pasetti M, Invernizzi C, Iora P (2014) Thermal stability of working fluids for organic Rankine cycles: an improved survey method and experimental results for cyclopentane, isopentane and n-butane. Appl Therm Eng 73(1):762-772

Peters F, Rodemann T (1998) Design and performance of a rapid piston expansion tube for the investigation of droplet condensation. Exp Fluids 24(4):300-307

Rinaldi E, Pecnik R, Colonna P (2015) Computational fluid dynamic simulation of a supercritical $\mathrm{CO}_{2}$ compressor performance map. J Eng Gas Turbines Power 137(7):1-7

Schrijer F, Bannink W (2010) Description and flow assessment of the delft hypersonic Ludwieg tube. J Spacecraft Rockets 47(1):125-133

Thompson PA (1971) A fundamental derivative in gasdynamics. Phys Fluids 14(9):1843-1849

Thompson PA (1988) Compressible fluid dynamics. McGraw-Hill, New York

Thompson PA, Loutrel WF (1973) Opening time of brittle shock-tube diaphragms for dense fluids. Rev Sci Instrum 44(9):1436-1437

Wagner JL, Beresh SJ, Kearney SP, Trott WM, Castaneda JN, Pruett BO, Baer MR (2012) A multiphase shock tube for shock wave interactions with dense particle fields. Exp Fluids 52(6):1507-1517

Weith T, Heberle F, Preißinger M, Brüggeman D (2014) Performance of siloxanes mixtures in a high-temperature organic Rankine cycle considering the heat transfer characteristics during evaporation. Energies 7:5548-5565

Zamfirescu C, Dincer I (2009) Performance investigation of hightemperature heat pumps with various BZT working fluids. Thermochim Acta 488(12):66-77 